\title{
Non-classical conditional probability and the quantum no-cloning theorem
}

\author{
Gerd Niestegge \\ Fraunhofer ESK, Hansastr. 32, 80686 München, German状
}

\begin{abstract}
The quantum mechanical no-cloning theorem for pure states is generalized and transfered to the quantum logics with a conditional probability calculus in a rather abstract, though simple and basic fashion without relying on a tensor product construction or finite dimension as required in other generalizations.
\end{abstract}

PACS numbers: 03.65.Ta, 03.67.-a

Keywords: quantum information and communication, foundations of quantum mechanics, generalized probabilistic theories

\section{Introduction}

A pioneering result with far-reaching consequences in quantum information and communication theory is the no-cloning theorem, stating that unknown pure quantum states cannot be copied unless they are orthogonal [12, 28 30]. An interesting generalization is the nobroadcasting theorem for mixed states [3]. Originally, both were proved in Hilbert space quantum mechanics, then extended to the $\mathrm{C}^{*}$-algebraic setting [11] and later to finite-dimensional generic probabilistic models [1, 2] and to quantum logics [20]. In the latter case, only universal cloning is impossible, while the cloning of a small set or pair of states can be ruled out in the other cases. Though these results preclude the perfect cloning, the approximate or imperfect cloning of quantum states remains possible 8, 10, 19]. In this paper, the (perfect) cloning of a small set or pair of states is considered in the setting of quantum logics with a conditional probability calculus 21, 22], including finite-dimensional as well as infinite-dimensional models.

A quantum logic $E$ is a purely algebraic structure for the quantum events (or propositions). It is quite common to use an orthomodular partially ordered set or lattice [4, 5, 17, 24]. States are then defined in the same way as the classical probability measures, and conditional probabilities are postulated to behave like the classical ones on compatible subsets of $E$. Note that a subset is called compatible if it is contained in another subset of $E$ forming a Boolean algebra (i.e., in a classical subsystem of E) [7]. Some quantum logics entail unique conditional probabilities, many others don't. The classical Boolean algebras and the Hilbert space quantum logic (consisting of the closed subspaces or, equivalently, the selfadjoint projection operators) do and, in the latter case, conditionalization becomes identical with the state transition of the Lüders - von Neumann (i.e., projective) quantum measurement process [21]. Therefore, the quantum logics with unique conditional probabilities can be regarded as a generalized mathematical model of projective quantum measurement. Examples that are neither classical

* gerd.niestegge@web.de
Boolean algebras nor Hilbert space quantum logics (nor sublogics of them) can be constructed using the exceptional real Jordan algebras [21, 22].

In this framework, a very special type of conditional probability emerges in the non-classical case [21, 22]. It describes the probability for the transition from a past event $e$ to a future event $f$, independently of any underlying state, and results from the algebraic structure of the quantum logic $E$. This probability exists only for certain event pairs $e$ and $f$. It exists for all events $f \in E$, if $e$ is a minimal event (atom) in $E$. The states resulting in this way are called atomic. They represent a generalization of the pure states in Hilbert space quantum mechanics.

After the early pioneering work by Birkhoff and von Neumann in 1936 6], quantum logics have been studied extensively between 1960 and 1995 [4, 5, 17, 18, 23 27]. Various forms of conditional probability have also been considered [4, 9, 13 16]. However, the quantum logics which possess unique conditional probabilities and particularly the special type of the state-independent conditional probability have not received any attention before the author's work 21, 22].

Considering such a quantum logic, this special type of conditional probability is used in the present paper to prove, in a very basic fashion, the generalized no-cloning theorem for atomic states. A tensor product construction as used in the other approaches is not required. Instead, the embedding of two copies of $E$, which shall be compatible with each other, in a larger quantum logic $L$ is sufficient.

The paper is organized as follows. The algebraic structure of the quantum logic is considered in section 2 . Section 3 then turns to states and briefly sketches the non-classical conditional probability calculus from Refs. [21, 22]. The main results are presented in sections 4 and 5 . The proof of the quantum mechanical no-cloning theorem rests upon the following two basic properties of the inner product in the Hilbert space: It is invariant under the unitary cloning transformation and multiplicative for the Hilbert space tensor product. These properties are transferred to the non-classical conditional probabilities in a certain way (Lemmas 1(a) and 2), which then allows to mimic the quantum mechanical proof for the generalized no-cloning theorem (Theorem 1). In section 6 , the ties to Hilbert space quantum mechanics are pointed out. 


\section{Compatibility in orthomodular partially ordered sets}

In quantum mechanics, the measurable quantities of a physical system are represented by observables. Most simple are those observables where only the two discrete values 0 and 1 are possible as measurement outcome; these observables are called events (or propositions) and are elements of a mathematical structure called quantum logic.

In this paper, a quantum logic shall be an orthomodular partially ordered set $E$ with the partial ordering $\leq$, the orthocomplementation ', the smallest element 0 and the largest element $\mathbb{I}[4,5,17,24]$. This means that the following conditions are satisfied by all $e, f \in E$ :

(A) $e \leq f$ implies $f^{\prime} \leq e^{\prime}$.

(B) $\left(e^{\prime}\right)^{\prime}=e$.

(C) $e \leq f^{\prime}$ implies $e \vee f$, the supremum of $e$ and $f$, exists.

(D) $e \vee e^{\prime}=\mathbb{I}$.

(E) $f \leq e$ implies $e=f \vee\left(e \wedge f^{\prime}\right)$. (orthomodular law)

Here, $e \wedge f$ denotes the infimum of $e$ and $f$, which exists iff $e^{\prime} \vee f^{\prime}$ exists. Two elements $e, f \in E$ are called orthogonal if $e \leq f^{\prime}$ or, equivalently, $f \leq e^{\prime}$. An element $e \neq 0$ in $E$ is called an atom if there is no element $f$ in $E$ with $f \leq e$ and $0 \neq f \neq e$.

The interpretation of this mathematical terminology is as follows: orthogonal events are exclusive, $e^{\prime}$ is the negation of $e$, and $e \vee f$ is the disjunction of the two exclusive events $e$ and $f$.

It is not assumed that $E$ is a lattice (in a lattice, there is a smallest upper bound $e \vee f$ and largest lower bound $e \wedge f$ for any two elements $e$ and $f)$. If $E$ were a distributive lattice (i.e., $e \wedge(f \vee g)=(e \wedge f) \vee(e \wedge g)$ for all $e, f, g \in E)$, it would become a Boolean lattice or Boolean algebra. The orthomodular law is a weakening of the distributivity law.

Classical probability theory uses Boolean lattices as mathematical structure for the random events, and it can be expected that those subsets of $E$, which are Boolean lattices, behave classically. Therefore, a subset $E_{0}$ of $E$ is called compatible if there is a Boolean lattice $B$ with $E_{0} \subseteq B \subseteq E$. Any subset with pairwise orthogonal element is compatible [7]. Two subsets $E_{1}$ and $E_{2}$ of $E$ are called compatible with each other if the union of any compatible subset of $E_{1}$ with any compatible subset of $E_{2}$ is a compatible subset of $E$. Note that this does not imply that $E_{1}$ or $E_{2}$ themselves are compatible subsets.

A subset of an orthomodular lattice is compatible if each pair of elements in this subset forms a compatible subset. However, the pairwise compatibility of the elements of a subset of an orthomodular partially ordered set does not any more imply the compatibility of this subset [7].

A quantum logical structure, which is more general than the orthomodular partially ordered sets, has been used in Refs. [21, 22]. This more general structure is sufficient when only compatible pairs of elements in the quantum logic are considered. However, compatible subsets with more than two elements will play an important role in this paper.

A quantum logic is a purely algebraic structure, unfurling its full potential only when its state space has some nice properties which shall be considered in the next section.

\section{Non-classical conditional probability}

The states on the orthomodular partially ordered set $E$ are the analogue of the probability measures in classical probability theory, and conditional probabilities can be defined similar to their classical prototype. A state $\rho$ allocates the probability $\rho(f)$ with $0 \leq \rho(f) \leq 1$ to each event $f \in E$, is additive for orthogonal events, and $\rho(\mathbb{I})=1$. It then follows that $\rho(f) \leq \rho(e)$ for any two events $e, f \in E$ with $f \leq e$.

The conditional probability of an event $f$ under another event $e$ is the updated probability for $f \in E$ after the outcome of a first measurement has been the event $e \in E$; it is denoted by $\rho(f \mid e)$. Mathematically, it is defined by the conditions that the map $E \ni f \rightarrow \rho(f \mid e)$ is a state on $E$ and that it coincides with the classical conditional probability for those $f$ which are compatible with $e$. The second condition is equivalent to the identity $\rho(f \mid e)=$ $\rho(f) / \rho(e)$ for all events $f \in E$ with $f \leq e$. It must be assumed that $\rho(e) \neq 0$.

However, among the orthomodular partially ordered sets, there are many where no states or no conditional probabilities exist, or where the conditional probabilities are ambiguous. It shall now be assumed for the remaining part of this paper that

(F) there is a state $\rho$ on $E$ with $\rho(e) \neq 0$ for each $e \in E$ with $e \neq 0$,

(G) $E$ possesses unique conditional probabilities, and

(H) the state space of $E$ is strong; i.e., if

$$
\begin{aligned}
& \{\rho \mid \rho \text { is a state with } \rho(f)=1\} \\
\subseteq & \{\rho \mid \rho \text { is a state with } \rho(e)=1\}
\end{aligned}
$$

holds for two events $e$ and $f$ in $E$, then $f \leq e$.

If $\rho$ is a state with $\rho(e)=1$ for some event $e \in E$, then $\rho(f \mid e)=\rho(f)$ for all $f \in E$. This follows from $(\mathrm{G})$.

For some event pairs $e$ and $f$ in $E$, the conditional probability does not depend on the underlying state; this means $\rho_{1}(f \mid e)=\rho_{2}(f \mid e)$ for all states $\rho_{1}$ and $\rho_{2}$ with $\rho_{1}(e) \neq 0 \neq \rho_{2}(e)$. This special conditional probability is then denoted by $\mathbb{P}(f \mid e)$. The following two conditions are equivalent for an event pair $e, f \in E$ :

(i) $\mathbb{P}(f \mid e)$ exists and $\mathbb{P}(f \mid e)=s$.

(ii) $\rho(e)=1$ implies $\rho(f)=s$ for the states $\rho$ on $E$. Due to condition (H), $f \leq e$ holds for two events $e$ and $f$ in $E$ if and only if $\mathbb{P}(e \mid f)=1$. Moreover, $e$ and $f$ are orthogonal if and only if $\mathbb{P}(e \mid f)=0$. 
$\mathbb{P}(f \mid e)$ exists for all $f \in E$ if and only if $e$ is an atom (minimal event), which results in the atomic state $\mathbb{P}_{e}$ defined by $\mathbb{P}_{e}(f):=\mathbb{P}(f \mid e)$. This is the unique state allocating the probability value 1 to the atom $e$. For two atoms $e$ and $f$ in $E$, the following four identities are equivalent: $\mathbb{P}_{e}(f)=1, \mathbb{P}_{f}(e)=1, \mathbb{P}_{e}=\mathbb{P}_{f}$, and $e=f$.

\section{Morphisms}

In this section, the invariance of the special conditional probability $\mathbb{P}(\cdot \mid \cdot)$ under quantum logical morphisms is studied. In the proof of the main result, this will later replace the invariance of the inner product under unitary transformations in the Hilbert space setting.

Suppose $E$ and $F$ are orthomodular partially ordered sets and $T: E \rightarrow F$ is an (algebraic) morphism (i.e., $T e_{1} \leq T e_{2}$ for $e_{1}, e_{2} \in E$ with $e_{1} \leq e_{2}, T\left(e^{\prime}\right)=(T e)^{\prime}$ for all $e \in E$ and $T \mathbb{I}=\mathbb{I}$ ). A dual transformation $T^{*}$, mapping the states $\rho$ on $F$ to states $T^{*} \rho$ on $E$, is then defined by $\left(T^{*} \rho\right)(e):=\rho(T e)$ for $e \in E$. In the case where both $E$ and $F$ possess unique conditional probabilities,

$$
\left(T^{*} \rho\right)\left(e_{2} \mid e_{1}\right)=\rho\left(T e_{2} \mid T e_{1}\right)
$$

holds for all events $e_{1}, e_{2} \in E$ with $\rho\left(T e_{1}\right) \neq 0$. To see this, consider the state $e \rightarrow \rho\left(T e \mid T e_{1}\right)$ on $E$; the uniqueness of the conditional probability implies that it must coincide with the state $e \rightarrow\left(T^{*} \rho\right)\left(e \mid e_{1}\right)$.

Lemma 1: Let $E$ and $F$ be orthomodular partially ordered sets, satisfying (F) and (G), and let $T: E \rightarrow F$ be a morphism.

(a) If $\mathbb{P}\left(e_{2} \mid e_{1}\right)$ exists for two events $e_{1}$ and $e_{2}$ in $E$ with $T e_{1} \neq 0$, then $\mathbb{P}\left(T e_{2} \mid T e_{1}\right)$ exists and

$$
\mathbb{P}\left(T e_{2} \mid T e_{1}\right)=\mathbb{P}\left(e_{2} \mid e_{1}\right)
$$

(b) If $T$ is an isomorphism, then $T^{*} \mathbb{P}_{f}=\mathbb{P}_{T^{-1} f}$ for the atoms $f$ in $F$.

Proof. (a) Suppose $\mathbb{P}\left(e_{2} \mid e_{1}\right)$ exists for $e_{1}$ and $e_{2}$ in $E$. Then $\mathbb{P}\left(e_{2} \mid e_{1}\right)=\left(T^{*} \rho\right)\left(e_{2} \mid e_{1}\right)=\rho\left(T e_{2} \mid T e_{1}\right)$ for all states $\rho$ on $F$ with $\left(T^{*} \rho\right)\left(e_{1}\right)=\rho\left(T e_{1}\right)>0$. Therefore, $\mathbb{P}\left(T e_{2} \mid T e_{1}\right)$ exists and is identical with $\mathbb{P}\left(e_{2} \mid e_{1}\right)$.

(b) Let $f$ be an atom in $F$. Then $T^{-1} f$ is an atom in $E$ and, by (a), $\mathbb{P}\left(e \mid T^{-1} f\right)=\mathbb{P}(T e \mid f)$ for $e \in E$. Therefore, $\mathbb{P}_{T^{-1} f}=T^{*} \mathbb{P}_{f}$.

\section{The generalized no-cloning theorem}

In this section, a quantum logic shall always be an orthomodular partially ordered set and shall satisfy $(\mathrm{F}),(\mathrm{G})$ and $(\mathrm{H})$. Suppose that $E$ is a quantum logic and that two copies of it are contained in the larger quantum logic $L$. This means that there are two injective morphisms $\pi_{1}: E \rightarrow L$ and $\pi_{2}: E \rightarrow L$. Moreover, suppose

(I) the subsets $\pi_{1}(E)$ and $\pi_{2}(E)$ of $L$ are compatible with each other and
(J) $\pi_{1}(e) \wedge \pi_{2}(f)$ is an atom in $L$ for each pair of atoms $e$ and $f$ in $E$.

The proof of the quantum mechanical no-cloning theorem rests upon the multiplicativity of the inner product for the Hilbert space tensor product. The following lemma provides the substitute for this in the more general setting.

Lemma 2: Suppose $\mathbb{P}\left(e_{2} \mid e_{1}\right)$ and $\mathbb{P}\left(f_{2} \mid f_{1}\right)$ both exist for $e_{1}, e_{2}, f_{1}, f_{2} \in E$. Then

$$
\mathbb{P}\left(\left(\pi_{1} e_{2}\right) \wedge\left(\pi_{2} f_{2}\right) \mid\left(\pi_{1} e_{1}\right) \wedge\left(\pi_{2} f_{1}\right)\right)
$$

exists and

$$
\mathbb{P}\left(\left(\pi_{1} e_{2}\right) \wedge\left(\pi_{2} f_{2}\right) \mid\left(\pi_{1} e_{1}\right) \wedge\left(\pi_{2} f_{1}\right)\right)=\mathbb{P}\left(e_{2} \mid e_{1}\right) \mathbb{P}\left(f_{2} \mid f_{1}\right)
$$

Proof. Given the above assumptions, consider a state $\rho$ on $L$ with

$$
\rho\left(\left(\pi_{1} e_{1}\right) \wedge\left(\pi_{2} f_{1}\right)\right)=1
$$

Then $\rho\left(\pi_{1} e_{1}\right)=1=\rho\left(\pi_{2} f_{1}\right)$. Now define two states $\mu_{1}$ and $\mu_{2}$ on $E$ by

$$
\mu_{1}(e):=\rho\left(\left(\pi_{1} e\right) \wedge\left(\pi_{2} f_{1}\right)\right) \text { and } \mu_{2}(e):=\rho\left(\left(\pi_{1} e\right)\right)
$$

for $e \in E$. Note that $\mu_{1}$ is a state due to (I). Since $\mu_{1}\left(e_{1}\right)=1=\mu_{2}\left(e_{1}\right)$ and $\mathbb{P}\left(e_{2} \mid e_{1}\right)$ exists, it follows that $\mathbb{P}\left(e_{2} \mid e_{1}\right)=\mu_{1}\left(e_{2}\right)=\mu_{2}\left(e_{2}\right)$ and $\mathbb{P}\left(e_{2}^{\prime} \mid e_{1}\right)=\mu_{1}\left(e_{2}^{\prime}\right)=$ $\mu_{2}\left(e_{2}^{\prime}\right)$. Thus

$$
\mathbb{P}\left(e_{2} \mid e_{1}\right)=\rho\left(\left(\pi_{1} e_{2}\right) \wedge\left(\pi_{2} f_{1}\right)\right)=\rho\left(\left(\pi_{1} e_{2}\right)\right)
$$

and

$$
\mathbb{P}\left(e_{2}{ }^{\prime} \mid e_{1}\right)=\rho\left(\left(\pi_{1} e_{2}{ }^{\prime}\right) \wedge\left(\pi_{2} f_{1}\right)\right)=\rho\left(\left(\pi_{1} e_{2}{ }^{\prime}\right)\right) .
$$

In the case $\mathbb{P}\left(e_{2} \mid e_{1}\right)>0$, define the state $\nu$ on $E$ by

$$
\nu(f):=\frac{\rho\left(\left(\pi_{1} e_{2}\right) \wedge\left(\pi_{2} f\right)\right)}{\mathbb{P}\left(e_{2} \mid e_{1}\right)}
$$

for $f \in E$. Then $\nu\left(f_{1}\right)=1$ and therefore

$$
\mathbb{P}\left(f_{2} \mid f_{1}\right)=\nu\left(f_{2}\right)=\frac{\rho\left(\left(\pi_{1} e_{2}\right) \wedge\left(\pi_{2} f_{2}\right)\right)}{\mathbb{P}\left(e_{2} \mid e_{1}\right)} .
$$

In the case $\mathbb{P}\left(e_{2} \mid e_{1}\right)=0$, it follows $\mathbb{P}\left(e_{2}{ }^{\prime} \mid e_{1}\right)=1$. Then $e_{1} \leq e_{2}{ }^{\prime}$ and $e_{2} \leq e_{1}^{\prime}$. Therefore,

$\rho\left(\left(\pi_{1} e_{2}\right) \wedge\left(\pi_{2} f_{2}\right)\right) \leq \rho\left(\pi_{1} e_{2}\right) \leq \rho\left(\pi_{1} e_{1}{ }^{\prime}\right)=1-\rho\left(\pi_{1} e_{1}\right)=0$

and $\rho\left(\left(\pi_{1} e_{2}\right) \wedge\left(\pi_{2} f_{2}\right)\right)=0$. In both cases,

$$
\rho\left(\left(\pi_{1} e_{2}\right) \wedge\left(\pi_{2} f_{2}\right)\right)=\mathbb{P}\left(e_{2} \mid e_{1}\right) \mathbb{P}\left(f_{2} \mid f_{1}\right) .
$$

Since this holds for all states $\rho$ on $L$ with $\rho\left(\left(\pi_{1} e_{1}\right) \wedge\right.$ $\left.\left(\pi_{2} f_{1}\right)\right)=1$, it finally follows that

$$
\mathbb{P}\left(\left(\pi_{1} e_{2}\right) \wedge\left(\pi_{2} f_{2}\right) \mid\left(\pi_{1} e_{1}\right) \wedge\left(\pi_{2} f_{1}\right)\right)=\mathbb{P}\left(e_{2} \mid e_{1}\right) \mathbb{P}\left(f_{2} \mid f_{1}\right) .
$$


Note that the proof of Lemma 2 does neither require assumption $(\mathrm{J})$ nor any tensor product construction, but instead only assumption(I).

A state $\rho$ on $L$ can be restricted to each one of the two copies of $E$ in $L$, resulting in the following two states on $E: \pi_{1}^{*} \rho=\rho \pi_{1}$ and $\pi_{2}^{*} \rho=\rho \pi_{2}$.

Lemma 3: Let $e$ and $f$ be atoms in $E$ and $\rho$ a state on $L$. Then $\rho \pi_{1}=\mathbb{P}_{e}$ and $\rho \pi_{2}=\mathbb{P}_{f}$ if and only if $\rho=$ $\mathbb{P}_{\left(\pi_{1} e\right) \wedge\left(\pi_{2} f\right)}$.

Proof. Assume $\rho \pi_{1}=\mathbb{P}_{e}$ and $\rho \pi_{2}=\mathbb{P}_{f}$. Then (I) implies

$$
1=\mathbb{P}_{e} e=\rho \pi_{1} e=\rho\left(\left(\pi_{1} e\right) \wedge\left(\pi_{2} f\right)\right)+\rho\left(\left(\pi_{1} e\right) \wedge\left(\pi_{2} f^{\prime}\right)\right)
$$

and

$$
0 \leq \rho\left(\left(\pi_{1} e\right) \wedge\left(\pi_{2} f^{\prime}\right)\right) \leq \rho \pi_{2} f^{\prime}=\mathbb{P}_{f} f^{\prime}=0 .
$$

Therefore $1=\rho\left(\left(\pi_{1} e\right) \wedge\left(\pi_{2} f\right)\right)$ and, since $\left(\pi_{1} e\right) \wedge\left(\pi_{2} f\right)$ is an atom in $L$,

$$
\rho=\mathbb{P}_{\left(\pi_{1} e\right) \wedge\left(\pi_{2} f\right)} .
$$

Now assume $\rho=\mathbb{P}_{\left(\pi_{1} e\right) \wedge\left(\pi_{2} f\right)}$ and $a \in E$. Then by Lemmas 1 (a) and 2

$$
\begin{aligned}
\rho \pi_{1} a & =\mathbb{P}\left(\left(\pi_{1} a\right) \mid\left(\pi_{1} e\right) \wedge\left(\pi_{2} f\right)\right) \\
& =\mathbb{P}\left(\left(\pi_{1} a\right) \wedge\left(\pi_{2} \mathbb{I}\right) \mid\left(\pi_{1} e\right) \wedge\left(\pi_{2} f\right)\right) \\
& =\mathbb{P}\left(\pi_{1} a \mid \pi_{1} e\right) \mathbb{P}\left(\pi_{2} \mathbb{I} \mid \pi_{2} f\right) \\
& =\mathbb{P}\left(\pi_{1} a \mid \pi_{1} e\right) \\
& =\mathbb{P}(a \mid e)=\mathbb{P}_{e} a
\end{aligned}
$$

The second identity $\rho \pi_{2}=\mathbb{P}_{f}$ follows in the same way.

Now suppose that $C$ is a set of atoms in $E$ and that $f$ is a fixed atom in $E$, that the local state on the first copy of $E$ is any element in $\left\{\mathbb{P}_{e} \mid e \in C\right\}$ and that the local state on the second copy of $E$ is $\mathbb{P}_{f}$. For the state $\rho$ on $L$ this means that $\rho \pi_{1}=\mathbb{P}_{e}$ for some unknown $e \in C$ and $\rho \pi_{2}=\mathbb{P}_{f}$.

In the usual quantum mechanical setting, the cloning is performed by a unitary transformation on the Hilbert space tensor product. In this paper, it shall be performed by an automorphism of $L$; cloning means that the automorphism transforms the initial local state on the second copy of $E$ to a copy of the unchanged local state on the first copy of $E$. After the transformation, both copies of $E$ are in the same local state and this is the local state on the first copy before the transformation.

Definition 1: A cloning transformation for $\left\{\mathbb{P}_{e} \mid e \in C\right\}$ is an automorphism $T$ of the quantum logic $L$ such that $\left(T^{*} \rho\right) \pi_{1}=\rho \pi_{1}=\left(T^{*} \rho\right) \pi_{2}$ holds for the states $\rho$ on $L$ with $\rho \pi_{1} \in\left\{\mathbb{P}_{e} \mid e \in C\right\}$ and $\rho \pi_{2}=\mathbb{P}_{f}$.

Theorem 1: A cloning transformation $T$ for $\left\{\mathbb{P}_{e} \mid e \in C\right\}$ exists only if the atoms in $C$ are pairwise orthogonal.

Proof. Assume $T$ is a cloning transformation for $\left\{\mathbb{P}_{e} \mid e \in C\right\}$. Note that, by Lemma $3, \rho \pi_{1}=\mathbb{P}_{e}$ and $\rho \pi_{2}=\mathbb{P}_{f}$ holds for the states $\rho=\mathbb{P}_{\left(\pi_{1} e\right) \wedge\left(\pi_{2} f\right)}$ with $e \in C$ and, furthermore, $\left(T^{*} \rho\right) \pi_{1}=\mathbb{P}_{e}=\left(T^{*} \rho\right) \pi_{2}$ with $e \in C$ implies $T^{*} \rho=\mathbb{P}_{\left(\pi_{1} e\right) \wedge\left(\pi_{2} e\right)}$. Therefore by Lemma $1(\mathrm{~b})$

$$
\mathbb{P}_{\left(\pi_{1} e\right) \wedge\left(\pi_{2} e\right)}=T^{*} \mathbb{P}_{\left(\pi_{1} e\right) \wedge\left(\pi_{2} f\right)}=\mathbb{P}_{T^{-1}\left(\left(\pi_{1} e\right) \wedge\left(\pi_{2} f\right)\right)}
$$

and thus

$$
T^{-1}\left(\left(\pi_{1} e\right) \wedge\left(\pi_{2} f\right)\right)=\left(\left(\pi_{1} e\right) \wedge\left(\pi_{2} e\right)\right)
$$

for each $e \in C$. Now assume $e_{1}, e_{2} \in C$ and consider

$$
\mathbb{P}\left(\left(\pi_{1} e_{2}\right) \wedge\left(\pi_{2} f\right) \mid\left(\pi_{1} e_{1}\right) \wedge\left(\pi_{2} f\right)\right) .
$$

On the one hand, the repeated application of Lemmas 1 (a) and 2 yields

$$
\begin{aligned}
\mathbb{P} & \left(\left(\pi_{1} e_{2}\right) \wedge\left(\pi_{2} f\right) \mid\left(\pi_{1} e_{1}\right) \wedge\left(\pi_{2} f\right)\right) \\
& =\mathbb{P}\left(T^{-1}\left(\left(\pi_{1} e_{2}\right) \wedge\left(\pi_{2} f\right)\right) \mid T^{-1}\left(\left(\pi_{1} e_{1}\right) \wedge\left(\pi_{2} f\right)\right)\right) \\
& =\mathbb{P}\left(\left(\pi_{1} e_{2}\right) \wedge\left(\pi_{2} e_{2}\right) \mid\left(\pi_{1} e_{1}\right) \wedge\left(\pi_{2} e_{1}\right)\right) \\
& =\mathbb{P}\left(\pi_{1} e_{2} \mid \pi_{1} e_{1}\right) \mathbb{P}\left(\pi_{2} e_{2} \mid \pi_{2} e_{1}\right) \\
& =\mathbb{P}\left(e_{2} \mid e_{1}\right) \mathbb{P}\left(e_{2} \mid e_{1}\right) \\
& =\left(\mathbb{P}\left(e_{2} \mid e_{1}\right)\right)^{2}
\end{aligned}
$$

and, on the other hand,

$$
\begin{aligned}
\mathbb{P} & \left(\left(\pi_{1} e_{2}\right) \wedge\left(\pi_{2} f\right) \mid\left(\pi_{1} e_{1}\right) \wedge\left(\pi_{2} f\right)\right) \\
& =\mathbb{P}\left(\pi_{1} e_{2} \mid \pi_{1} e_{1}\right) \mathbb{P}\left(\pi_{2} f \mid \pi_{2} f\right) \\
& \left.=\mathbb{P}\left(e_{2} \mid e_{1}\right) \mathbb{P}(f \mid f)\right) \\
& =\mathbb{P}\left(e_{2} \mid e_{1}\right) .
\end{aligned}
$$

Therefore, $\left(\mathbb{P}\left(e_{2} \mid e_{1}\right)\right)^{2}=\mathbb{P}\left(e_{2} \mid e_{1}\right)$ and $\mathbb{P}\left(e_{2} \mid e_{1}\right) \in\{0,1\}$. This means that $e_{1}$ and $e_{2}$ are either orthogonal or identical.

If $L$ is a finite Boolean algebra (i.e., classical), different atoms are orthogonal and a cloning transformation $T$ is defined by extending to $L$ the following permutation of the atoms in $L: T\left(\left(\pi_{1} e\right) \wedge\left(\pi_{2} e\right)\right)=\left(\pi_{1} e\right) \wedge\left(\pi_{2} f\right)$ and $T\left(\left(\pi_{1} e\right) \wedge\left(\pi_{2} f\right)\right)=\left(\pi_{1} e\right) \wedge\left(\pi_{2} e\right)$ for all $e \in C, T d=d$ for the other atoms $d$ in $L$. However, non-orthogonal atoms are quite characteristic of quantum mechanics and Theorem 1 rules out that the corresponding atomic states can be cloned.

\section{Quantum mechanics}

Quantum mechanics uses a special quantum logic; it consists of the self-adjoint projection operators on a Hilbert space $H$ and is an orthomodular lattice. Compatibility here means that the self-adjoint projection operators commute. Conditions $(\mathrm{F})$ and $(\mathrm{H})$ in section 3 are satisfied, and the unique conditional probabilities exist $(\mathrm{G})$ unless the dimension of $H$ is two 21]. Moreover, it has been shown in Ref. 21] that, with two self-adjoint projection operators $e$ and $f$ on $H$, the conditional probability has the shape

$$
\rho(f \mid e)=\frac{\operatorname{trace}(\text { aefe })}{\operatorname{trace}(a e)}=\frac{\operatorname{trace}(\text { eaef })}{\operatorname{trace}(\text { ae })}
$$


for a state $\rho$ defined by the statistical operator $a$ (i.e., $a$ is a self-adjoint operator on $H$ with non-negative spectrum and $\operatorname{trace}(a)=1)$. The above identity reveals that conditionalization becomes identical with the state transition of the Lüders - von Neumann measurement process. Therefore, the conditional probabilities defined in section 3 can be regarded as a generalized mathematical model of projective quantum measurement.

$\mathbb{P}(f \mid e)$ exists with $\mathbb{P}(f \mid e)=s$ if and only if the operators $e$ and $f$ satisfy the algebraic identity efe $=$ se. This transition probability between the outcomes of two consecutive measurements is independent of any underlying state and results from the algebraic structure of the quantum logic.

The atoms are the self-adjoint projections on the onedimensional subspaces of $H$; if $e$ is an atom and $\xi$ a normalized vector in the corresponding one-dimensional subspace, then $\mathbb{P}(f \mid e)=\langle\xi \mid f \xi\rangle$. The atomic states thus coincide with the quantum mechanical pure states or vector states. Their general non-orthogonality is quite characteristic of quantum mechanics.

The quantum mechanical model of a composite system consisting of two copies is the Hilbert space tensor product $H \otimes H$. The self-adjoint projection operators $e$ on $H$ are mapped to two copies on $H \otimes H$ by $\pi_{1}(e):=e \otimes \mathbb{I}$ and $\pi_{2}(e):=\mathbb{I} \otimes e$. Note that $(\mathrm{I})$ and $(\mathrm{J})$ are then satisfied. Time evolutions of the composite system are described by unitary transformations of $H \otimes H$. Therefore, the cloning operation should be such a transformation. It defines an automorphism of the quantum logic of $H \otimes H$.

Theorem 1 thus includes the quantum mechanical nocloning theorem for pure states as a special case. Instead of the Hilbert space and tensor product formalism, Theorem 1 requires only a few very basic principles; these are the existence and the uniqueness of the conditional probabilities and the existence of two compatible copies of the system in a larger system. Nevertheless, the proof of the no-cloning theorem in the quantum mechanical Hilbert space formalism can be mimicked, replacing the Hilbert space inner product $\langle\mid\rangle$ by the specific stateindependent conditional probability $\mathbb{P}(\mid)$.

Theorem 1 considers only the atomic or pure states, while other approaches to a generalized no-cloning or no broadcasting theorem [1, 2, 20] include the mixed states. On the other hand, these approaches are restricted to finite-dimensional theories or universal cloning and need an explicit tensor product construction.
[1] H. Barnum, J. Barrett, M. Leifer, and A. Wilce. Cloning and broadcasting in generic probabilistic theories. arXiv:quant-ph/0611295, 2006.

[2] H. Barnum, J. Barrett, M. Leifer, and A. Wilce. Generalized no-broadcasting theorem. Physical review letters, 99(24):240501, 2007.

[3] H. Barnum, C. M. Caves, C. A. Fuchs, R. Jozsa, and B. Schumacher. Noncommuting mixed states cannot be broadcast. Physical Review Letters, 76(15):2818, 1996.

[4] E. G. Beltrametti, G. Cassinelli, and G.-C. Rota. The logic of quantum mechanics. Cambridge University Press, 1984.

[5] L. Beran. Orthomodular lattices. Springer, 1985.

[6] G. Birkhoff and J. von Neumann. The logic of quantum mechanics. Annals of Mathematics, 37:823-843, 1936.

[7] J. Brabec. Compatibility in orthomodular posets. Časopis pro pěstování matematiky, 104(2):149-153, 1979.

[8] D. Bruß, D. P. DiVincenzo, A. Ekert, C. A. Fuchs, C. Macchiavello, and J. A. Smolin. Optimal universal and state-dependent quantum cloning. Phys. Rev. A, 57:2368-2378, Apr 1998.

[9] J. Bub. Von Neumann's projection postulate as a probability conditionalization rule in quantum mechanics. Journal of Philosophical Logic, 6(1):381-390, 1977.

[10] V. Bužek and M. Hillery. Quantum copying: Beyond the no-cloning theorem. Physical Review A, 54(3):1844, 1996.

[11] R. Clifton, J. Bub, and H. Halvorson. Characterizing quantum theory in terms of information-theoretic constraints. Foundations of Physics, 33(11):1561-1591, 2003.

[12] D. Dieks. Communication by EPR devices. Physics Letters A, 92(6):271-272, 1982.

[13] C. M. Edwards and G. T. Rüttimann. On condi- tional probability in GL spaces. Foundations of Physics, 20(7):859-872, 1990.

[14] M. Friedman and H. Putnam. Quantum logic, conditional probability, and interference. Dialectica, 32(34):305-315, 1978.

[15] J. Gunson. On the algebraic structure of quantum mechanics. Communications in Mathematical Physics, 6(4):262-285, 1967.

[16] W. Guz. Conditional probability and the axiomatic structure of quantum mechanics. Fortschritte der Physik, 29(8):345-379, 1981.

[17] G. Kalmbach. Orthomodular Lattices. Academic Press, London, 1983.

[18] H. A. Keller. Ein nicht-klassischer Hilbertscher Raum. Mathematische Zeitschrift, 172(1):41-49, 1980.

[19] Y. Kitajima. Imperfect cloning operations in algebraic quantum theory. Foundations of Physics, 45(1):62-74, 2015.

[20] T. Miyadera and H. Imai. No-cloning theorem on quantum logics. Journal of Mathematical Physics, 50(10):-, 2009.

[21] G. Niestegge. Non-Boolean probabilities and quantum measurement. Journal of Physics A: Mathematical and General, 34(30):6031, 2001.

[22] G. Niestegge. An approach to quantum mechanics via conditional probabilities. Foundations of Physics, 38(3):241-256, 2008.

[23] C. Piron. Axiomatique quantique. Helvetica physica acta, 37(4-5):439-468, 1964.

[24] P. Pták and S. Pulmannová. Orthomodular structures as quantum logics. Kluwer, Dordrecht, 1991.

[25] M. P. Soler. Characterization of Hilbert spaces by orthomodular spaces. Communications in Algebra, 23(1):219- 
$243,1995$.

[26] V. S. Varadarajan. Geometry of Quantum Theory, Vol. 1. Van Nostrand - Reinhold, New York, 1968.

[27] V. S. Varadarajan. Geometry of Quantum Theory, Vol. 2. Van Nostrand - Reinhold, New York, 1970.

[28] R. F. Werner. Quantum information theory - an invitation. In Quantum information, pages 14-57. Springer,
2001.

[29] W. K. Wootters and W. H. Zurek. A single quantum cannot be cloned. Nature, 299(5886):802-803, 1982.

[30] H. P. Yuen. Amplification of quantum states and noiseless photon amplifiers. Physics Letters A, 113(8):405407, 1986. 